\title{
Does Decision-Making Speed Depend on Non-interactive Others?
}

\author{
Atsuko Inoue and Atsunori Ariga
}

\begin{abstract}
As it is experienced in daily life, people are susceptible to others' behaviors, choices, and their mere presence in many ways, even when they are non-interactive. Several studies on consumer behavior have reported this phenomenon. These studies, however, focused on how decision-making outcomes are affected by others, whereas none have examined effects on decision-making speed. Therefore, we investigated the effect of non-interactive others on decision-making speed.

Two laboratory experiments were conducted. In experiment 1, 96 university students were randomly assigned four conditions which include (1) subject by him/ herself, with other person performing task with (2) normal/(3) fast/(4) slow speed. In experiment 2, another fast condition added. Under this condition, subjects performed task with fast speed sound. Stimuli were 18 pairs of images, each member of which was derived from the same category. Both experiments were computer based.

The result showed that speed contagion occurred in fast speed condition. But in case of slow it didn't happen. Interestingly, there were no differences of satisfaction for the decision between four conditions. We also found this speed contagion happened only when there is physical presence of other. That means the only fast speed sound cannot make it happen. Because decision-making during a purchase is most frequently made in others' presence, our research would contribute to understanding decision-making under realistic social contexts.
\end{abstract}

\footnotetext{
A. Inoue $(\square)$

Seikei University, Tokyo, Japan

e-mail: ainoue@econ.seikei.ac.jp

\author{
A. Ariga \\ Rissho University, Tokyo, Japan \\ e-mail: ariga@ris.ac.jp
}

L. Petruzzellis, R.S. Winer (eds.), Rediscovering the Essentiality of Marketing, Developments in Marketing Science: Proceedings of the Academy of Marketing Science, DOI 10.1007/978-3-319-29877-1_15 